\title{
Toripalimab Used on the Same Day or Third Day With Chemotherapy for Neoadjuvant Chemoimmunotherapy in Locally Advanced Esophageal Squamous Cell Cancer-A Phase II Study
}

\section{Wenqun Xing}

Affiliated Tumor Hospital of Zhengzhou University: Henan Cancer Hospital

\section{Lingdi Zhao}

Affiliated Tumor Hospital of Zhengzhou University: Henan Cancer Hospital

\section{Yan Zheng}

Affiliated Tumor Hospital of Zhengzhou University: Henan Cancer Hospital

\section{Baoxing Liu}

Affiliated Tumor Hospital of Zhengzhou University: Henan Cancer Hospital

Xianben Liu

Affiliated Tumor Hospital of Zhengzhou University: Henan Cancer Hospital

\section{Tiepeng Li}

Affiliated Tumor Hospital of Zhengzhou University: Henan Cancer Hospital

Yong Zhang

Affiliated Tumor Hospital of Zhengzhou University: Henan Cancer Hospital

\section{Baozhen Ma}

Affiliated Tumor Hospital of Zhengzhou University: Henan Cancer Hospital

\section{Yonghao Yang}

Affiliated Tumor Hospital of Zhengzhou University: Henan Cancer Hospital

\section{Yiman Shang}

Affiliated Tumor Hospital of Zhengzhou University: Henan Cancer Hospital

\section{Xiaomin Fu}

Affiliated Tumor Hospital of Zhengzhou University: Henan Cancer Hospital

\section{Guanghui Liang}

Affiliated Tumor Hospital of Zhengzhou University: Henan Cancer Hospital

\section{Dongfeng Yuan}

Affiliated Tumor Hospital of Zhengzhou University: Henan Cancer Hospital Jinrong Qu

Affiliated Tumor Hospital of Zhengzhou University: Henan Cancer Hospital 


\section{Xiaofei Chai}

Affiliated Tumor Hospital of Zhengzhou University: Henan Cancer Hospital

\section{He Zhang}

Affiliated Tumor Hospital of Zhengzhou University: Henan Cancer Hospital

\section{Zibing Wang}

Affiliated Tumor Hospital of Zhengzhou University: Henan Cancer Hospital

\section{Hongwei Lin}

Affiliated Tumor Hospital of Zhengzhou University: Henan Cancer Hospital

\section{Liang Liu}

Tianjin Tumor Hospital

\section{Xiubao Ren}

Tianjin Tumor Hospital

\section{Jiangong Zhang}

Affiliated Tumor Hospital of Zhengzhou University: Henan Cancer Hospital

Quanli Gao ( $\sim$ gaoquanli2015@126.com )

Affiliated Cancer Hospital of Zhengzhou University \& Henan Cancer Hospital

\section{Research}

Keywords: toripalimab, chemoimmunotherapy, sequence, esophageal squamous cell carcinoma, pathological complete response

Posted Date: September 17th, 2021

DOI: https://doi.org/10.21203/rs.3.rs-877899/v1

License: (c) (1) This work is licensed under a Creative Commons Attribution 4.0 International License. Read Full License 


\section{Abstract \\ Background}

There is no standard neoadjuvant therapy for locally advanced esophageal cancer in China. The role of neoadjuvant chemotherapy plus immunotherapy for locally advanced esophageal cancer is still being explored.

\section{Methods}

This open-label, randomized phase II study was conducted at a single center between July 2019 and September 2020, 30 patients with locally advanced ESCC (T3, T4, or lymph-node positive) were enrolled. Patients were randomized according to the enrollment order at a 1:1 ratio to receive chemotherapy on day 1 and toripalimab on day 3 (experiment group) or chemotherapy and toripalimab on day 1 (control group). The chemotherapeutic regimen was paclitaxel and cisplatin. Surgery was performed 4 to 6 weeks after the second cycle of chemoimmunotherapy. The primary endpoint was pCR rate, the secondary endpoint were safety and disease-free survival.

\section{Results}

Thirty patients completed at least one cycle of chemoimmunotherapy; 11 in the experimental group and 13 in the control group received surgery. R0 resection was got in all these 24 patients. Four patients (36\%) in the experimental group and one $(7 \%)$ in the control group achieved pCR. The experimental group showed a statistically non-significant higher pCR rate $(P=0.079)$. PD-L1 combined positive score (CPS) examination was performed in 14 patients; one in control group had a PD-L1 CPS of 10 and PCR was achieved, while the left 13 were all $\leq 1,11$ of the 13 patients received surgery in which two (in the experimental group) got $\mathrm{pCR}$. Two patients endured from $\geq$ grade 3 adverse events, one suffered from grade 3 immune-related enteritis after one cycle of chemoimmunotherapy and dropped off the study. Another patient died from severe pulmonary infection and troponin elevation after surgery.

\section{CONCLUSIONS}

Although the primary endpoint was not met, initial results of this study showed that delaying toripalimab to day 3 in chemoimmunotherapy might achieve a higher PCR rate than that on same day, and further large-sample clinical trials are needed to verify this.

\section{Trial registration:}

NCT 03985670, registered 10 June 2019. 


\section{Background}

Esophageal squamous cell cancer (ESCC) is one of the common malignancies which threaten the health and life of human beings, especially for the Chinese[1]. The standard treatment for patients at early stage is radical surgery. Patients with locally advanced ESCC have a high rate of recurrence. At present the standard treatment for locally advanced ESCC is simultaneous chemoradiotherapy[2, 3]. As the race differences between the East and West, neoadjuvant chemotherapy is usually applied in Japan and China[4, 5]. Immune checkpoint inhibitor represented by anti-PD-1 antibody has become one of the important regimens in advanced malignancies. Anti-PD-1 antibody plus chemotherapy could prolong the survival time of patients with advanced ESCC and became the standard treatment for advanced ESCC[6].

Previous studies have shown a synergistic efficacy of chemotherapy and anti-PD-1 antibodies. Chemotherapy could induce immunogenic tumor cell death, deplete myeloid immunosuppressive cells selectively and lymphopenia, which reduces regulatory $T$ (Treg) cells and makes room for proliferation of effect T cells[7]. Thus, the combined application of these two agents has synergistic effects. Presently, chemoimmunotherapy combnations have comprised merely of standard-of-care chemotherapy regimens being added to immunotherapy and then being compared with chemotherapy plus placebo, the elements in this combination have not been optimized. For example a randomized phase III study concluded that the addition of chemotherapy to pembrolizumab worsened survival over pembrolizumab or chemotherapy as a single treatment in patients with PD-L1 CPS $\otimes 10$ [8]. Simultaneous administration of anti-PD-1 antibody and anti-OX40 antibody could reduce the anti-tumor effect of the anti-OX40 antibody, in contrast, administration of anti-PD-1 antibody after anti-OX40 antibody could produce synergistic antitumor effects in animal models, indicating the importance of the administrated sequence of anti-PD-1 antibody in combined cancer immunotherapy[9]. Theoretically, the sequence of anti-PD-1 antibodies and chemotherapeutic agents administrated may also affect the efficacy. PD-1 blockade may result in the expansion of tumor-specific T cells[10]; proliferating cells are more likely to be killed by chemotherapeutic drugs, so it may reduce the possibility of chemotherapy drugs to kill T cells by administrated anti-PD-1 antibodies after chemotherapy drugs. Therefore, it's imperative to explore the administrated sequence of anti-PD-1 antibodies and chemotherapeutic agents. Thus, we designed this study to explore the sequence of chemotherapy and toripalimab on the pCR rate and safety in patients with locally advanced ESCC.

Previous studies have shown a synergistic efficacy of chemotherapy and anti-PD-1 antibodies. Chemotherapy could induce immunogenictumor cell death, deplete myeloid immunosuppressive cells selectively and lymphopenia, which reduces regulatory $T$ (Treg) cells and makes room for proliferation of effect T cell[7]. Thus, the combined application of these two agents has synergistic effects. Presently, chemoimmunotherapy combnations have comprised merely of standard-of-care chemotherapy regimens being added to immunotherapy and then being compared with chemotherapy plus placebo, the elements in this combination have not been optimized. For example a randomized phase III study concluded that the addition of chemotherapy to pembrolizumab worsened survival over pembrolizumab or chemotherapy as a single treatment[8]. Simultaneous administration of anti-PD-1 antibody and anti-OX40 antibody could reduce the anti-tumor effect of the anti-OX40 antibody, in contrast, administration of anti- 
PD-1 antibody after anti-OX40 antibody could produce synergistic anti-tumor effects in animal models, indicating the importance of the administrated sequence of anti-PD-1 antibody in combined cancer immunotherapy[9]. Theoretically, the sequence of anti-PD-1 antibodies and chemotherapeutic agents administrated may also affect the efficacy. PD-1 blockade may result in the expansion of tumor-specific T cells[10]; proliferating cells are more likely to be killed by chemotherapeutic drugs, so it may reduce the possibility of chemotherapy drugs to kill T cells by administrated anti-PD-1 antibodies after chemotherapy drugs have been metabolized in the body. Therefore, it's imperative to explore the administrated sequence of anti-PD-1 antibodies and chemotherapeutic agents. Thus, we designed this study to explore the safety, efficacy and the sequence of chemotherapeutic agents and anti-PD- 1 agents in patients with locally advanced ESCC.

\section{Patients And Methods}

This study was approved by the Ethic Committee of Henan Cancer Hospital and was conducted in accordance with the Helsinki Declaration and Good Clinical Practice Guidelines. All enrolled patients provided written informed consent.

\section{Patients}

This was a randomized, open-label, single-center, phase II study. The eligibility criteria for patients eligibility were thoracic esophageal squamous cell carcinoma confirmed by pathology, without distant metastasis, resectable or potentially resectable assessed by thoracic surgical experts, ECOG 0-1, clinical stage II/III/IVa, age 18-70 years, adequate organ function, and no history of other malignant tumors. The exclusion criteria were severe infection within 4 weeks before enrollment, bronchial asthma requiring intermittent use of bronchodilators or medical intervention, use of immunosuppressants for coexisting diseases, obvious cardio-cerebrovascular diseases, severe allergic constitution, severe mental disorders, abnormal coagulation function, pulmonary fibrosis, interstitial pneumonia, pneumoconiosis, pregnant or lactating women, and not meeting the criteria for enrollment evaluated by investigators.

\section{Pretreatment stage}

All patients underwent thoracoabdominal and pelvic enhanced computed tomography (CT), esophageal enhanced MRI and endoscopic ultrasound for accurate clinical staging. Endoscopic ultrasound was used for the T stage. Enhanced (MRI) was used for the $\mathrm{N}$ stage and for measuring the length of the diseased esophagus in a diffusion-weighted imaging (DWI) sequence[11].

\section{Neoadjuvant therapy}

Patients were enrolled by investigators and randomly assigned (1:1) according to the enrollment order. Toripalimab (a type of anti-PD-1 antibody produced by Suzhou Zhonghe Biomedical Technology Co., Ltd., Suzhou, China) was administered at a fixed dose of $240 \mathrm{mg}$ every cycle. Paclitaxel was administered at a dose of $150-175 \mathrm{mg} / \mathrm{m}^{2}$ every cycle and cisplatin was administered at a dose of $70-75 \mathrm{mg} / \mathrm{m}^{2}$. Neither the investigators nor the patients were masked to treatment allocation. The patients in the experimental 
group received paclitaxel and cisplatin on day 1 and toripalimab on day 3 . The patients in the control group received paclitaxel and cisplatin and toripalimab on day 1 . The treatment cycle was 21 days in the control group and 21-28 days in the experimental group. Radical surgery was performed 4-6 weeks after the second chemotherapy plus toripalimab.

\section{Surgery}

McKeown esophagogastrectomy or thoracoscopic McKeown esophagogastrectomy was performed for upper and middle thoracic esophageal cancer, and Ivor Lewis esophagogastrectomy or thoracoscopic Ivor Lewis esophagogastric resection was used for middle and lower esophageal cancer.

\section{Pathological evaluation}

The depth of tumor invasion, lymph node metastasis, and surgical margin were evaluated according to the American Joint Committee on Cancer Criteria for esophageal carcinoma[12]. The extent of the residual tumors was divided into four categories: grade 0 , no evidence of viable tumor cells (pathologic complete response, pCR); grade 1, single cells or rare small groups of cancer cells (near complete response); grade 2, residual cancer cells with evident tumor regression, but more than single cells or rare small groups of cancer cells (partial response); and grade 3, extensive residual cancer without evident tumor regression (poor or no response)[13]. The postoperative pathological evaluation was carried out by two experienced pathologists. The expression of PD-L1 was examined using the Dako 22C3 antibody and counted based on a CPS.

\section{Objectives and endpoints}

The primary objective of this study was to explore the influence of sequence of toripalimab and chemotherapy on pCR rate in locally advanced ESCC. The secondary objectives were to explore the safety of chemotherapy and toripalimab as neoadjuvant therapy and the treatment related adverse events (AEs), which were evaluated according to the National Cancer Institute Common Terminology Criteria for Adverse Events Version 5.0 (NCI-CTC AE 5.0), and the influence of PD-L1 expression on PCR.

\section{Statistics}

As an exploratory observational study, a necessary sample size of 30 patients was calculated. The patients were divided into two groups according to their enrollment order. Continuous variables were calculated as means with standard deviation or medians with ranges. Categorical variables were calculated as frequencies. A t-test was performed to compare continuous variables, and a Chi-square test was performed for categorical variables. A two-sided $P$-value of $<0.05$ was considered statistically significant. All statistical analyses were performed using SPSS 22.0 (IBM Corp., Armonk, NY, USA).

\section{Results}

\section{Patients}


Thirty patients were enrolled between July 2019 and October 2020. The CONSORT reporting procedures are presented in Supplementary Table 1. The baseline characteristics of the patients are listed in Table 1. After neoadjuvant therapy, 24 patients underwent surgery. The flowchart of the study is presented in Fig. 1.

Table 1

Baseline characteristics of different groups

\begin{tabular}{|c|c|c|c|c|}
\hline \multirow[t]{2}{*}{ Characteristics } & \multicolumn{2}{|l|}{ Group } & \multirow[t]{2}{*}{ Statistics } & \multirow[t]{2}{*}{$P$ value } \\
\hline & Experimental group $(\mathrm{N}=15)$ & Control group(N = 15) & & \\
\hline Age(year) & $63.80 \pm 6.07$ & $63.13 \pm 5.05$ & $F=0.33$ & 0.746 \\
\hline Gender, $n(\%)$ & & & $\chi^{2}=2.73$ & 0.215 \\
\hline Male & $13(86.67 \%)$ & $9(60.00 \%)$ & & \\
\hline Female & $2(13.33 \%)$ & $6(40.00 \%)$ & & \\
\hline $\mathrm{T}$ & & & $\chi^{2}=2.73$ & 0.215 \\
\hline 3 & $9(60.00 \%)$ & $13(86.67 \%)$ & & \\
\hline 4 & $6(40.00 \%)$ & $2(13.33 \%)$ & & \\
\hline$N$ & & & $x^{2}=0.286$ & 0.867 \\
\hline 0 & $4(26.67 \%)$ & $3(20.00 \%)$ & & \\
\hline 1 & $8(53.33 \%)$ & $8(53.33 \%)$ & & \\
\hline 2 & $3(20.00 \%)$ & $4(26.67 \%)$ & & \\
\hline Stage & & & $\chi^{2}=1.815$ & 0.404 \\
\hline II & $3(20.00 \%)$ & $3(20.00 \%)$ & & \\
\hline III & $7(46.67 \%)$ & $10(66.67 \%)$ & & \\
\hline IVa & $5(33.33 \%)$ & $2(13.33 \%)$ & & \\
\hline ECOG PS & & & $\chi^{2}=1.22$ & 0.462 \\
\hline 0 & $8(53.33 \%)$ & $5(33.33 \%)$ & & \\
\hline 1 & $7(46.67 \%)$ & $10(66.66 \%)$ & & \\
\hline
\end{tabular}

\section{Efficacy}


Among the 30 patients, 2 did not undergo radiological evaluation and surgery after two neoadjuvant therapy cycles because of the COVID-19 pandemic, 1 patient withdrew from the study because of immune-associated enteritis. The radiological efficacy of neoadjuvant therapy was evaluated in the remaining 27 patients. The RECIST objective response rate (ORR) was 66.7\% (18/27 patients). Supplementary Fig. 1 showed the tumor burden changes from baseline.

Eleven patients in the experimental group and 13 patients in the control group underwent radical resection. R0 resection was got in all these 24 patients. Five patients $(20.8 \%)$ achieved pCR, four patients in the experimental group and one patient in the control group achieved pCR. The difference in $\mathrm{pCR}$ between the two groups was not statistically significant $\left(\chi^{2}=3.092, P=0.079\right)$. Neoadjuvant chemotherapy plus toripalimab did not increase the difficulty in surgery, and the median number of lymph nodes excised was 23(range 15-49). The numbers of resected lymph nodes were 21 and 26 in the experimental group and control group $(F=0.669, P=0.422)$. Supplementary Fig. 2 shows the esophageal lesions on MR of patients with or without PCR before and after neoadjuvant therapy in the two groups.

PD-L1 CPS examinations were performed with the tissue samples of 14 patients (7 in each group) whose tissue was enough before surgery. Only one patient in the control group had a PD-L1 CPS of 10, while the others had PD-L1 CPS $\leq 1$. The patient with PD-L1 CPS of 10 achieved PCR. Two of the six patients with PD-L1 CPS $\leq 1$ in the experiment group achieved pCR, while none of the six patients with PD-L1 CPS $\leq 1$ in the control group achieved pCR. Figure 2 shows the expression of PD-L1 CPS with PCR in both groups and the according to pathological appearance before therapy and after surgery. Figure 3 shows the expression of PD-L1 CPS with non-pCR in both groups and the according to pathological appearance before therapy and after surgery.

\section{Safety}

During the period of neoadjuvant therapy, almost all the patients developed treatment-related AEs. The most common AEs were nausea, vomiting, and peripheral neurotoxicity, which can be attributed to cisplatin and paclitaxel. Notably, one patient in the experimental group developed grade 3 immuneassociated enteritis after one cycle of therapy, this patient recovered after glucocorticoid and plasma exchange therapy. Postoperative pulmonary infection occurred in 13 patients, during which three developed sepsis and needed application of broad-spectrum antibiotics, two of the patients recovered from sepsis and one died 20 days after surgery. The patient who died from sepsis developed tropoin elevation which was suspected immune-related myocarditis during the course of sepsis. Anastomotic fistula occurred in two patients, one in the experiment group and one in the control group, both the two patients recovered from anastomotic fistula and neither of them underwent secondary surgery because of anastomotic fistula. The detailed treatment-related AEs and postoperative complications are shown in Table 2. There was no difference in treatment-related AEs and postoperative complications between the two groups. 
Table 2

Treatment-related adverse events and postoperative complications

\begin{tabular}{|c|c|c|c|c|c|c|}
\hline \multirow[t]{2}{*}{ Characteristics } & \multicolumn{2}{|c|}{ Experimental group } & \multicolumn{2}{|c|}{ Control group } & \multirow[t]{2}{*}{$\chi^{2}$} & \multirow[t]{2}{*}{$P$ value } \\
\hline & Grade $1 / 2$ & Grade $3 / 5$ & Grade $1 / 2$ & Grade $3 / 5$ & & \\
\hline Nausea and vomiting & 12 & & 15 & & 3.330 & 0.068 \\
\hline Diarrhea & 2 & & 3 & & 0.240 & 0.624 \\
\hline Mouth ulcers & 1 & & & & 1.034 & 1.034 \\
\hline Fatigue & 1 & & & & 1.034 & 0.309 \\
\hline Dizzy & 1 & & & & 1.034 & 0.309 \\
\hline Neuromuscular toxicity & 3 & & 5 & & 0.682 & 0.409 \\
\hline Leukopenia & 4 & & 4 & 2 & 0.600 & 0.439 \\
\hline Thrombocytopenia & 3 & & 1 & & 1.154 & 0.283 \\
\hline Elevated transaminase & & & 1 & & 1.034 & 0.309 \\
\hline Hypoglycemia & & & 1 & & 1.034 & 0.309 \\
\hline Hypokalemia & 2 & 1 & 1 & 3 & 0.186 & 0.666 \\
\hline Hyponatremia & 1 & & & 1 & 0.000 & 1.000 \\
\hline Hypocalcemia & & & 1 & & 1.034 & 0.309 \\
\hline Immune-related enteritis & & 1 & & & 1.034 & 0.309 \\
\hline $\begin{array}{l}\text { Immune-related } \\
\text { myocarditis }\end{array}$ & & & 1 & & 1.034 & 0.309 \\
\hline Pulmonary infection & 4 & 1 & 7 & 1 & 1.22 & 0.269 \\
\hline Anastomotic fistula & 1 & & 1 & & 0.000 & 1.000 \\
\hline
\end{tabular}

\section{Discussion}

To the best of our knowledge, in this study, we present for the first time that the sequence of chemotherapy and toripalimab might influence the PCR in patients with locally advanced ESCC, with relatively manageable treatment-related AEs. The pCR was $36 \%$ in the experimental group and $7 \%$ in the control group, suggesting that the different sequence of administration of chemotherapy and toripalimab might affect the clinical efficacy. The regimen was well-tolerated, and the AEs of neoadjuvant chemoimmunotherapy were similar to those reported in the studies on advanced stage esophageal cancer $[6,14]$. No new AEs occurred during the treatment. The most common AEs in the stage of neoadjuvant therapy were mainly caused by chemotherapeutic agents. One patient developed tropoin elevation during pulmonary infection after surgery, and this patient died from severe pulmonary infection. 
One patient withdrew from the study because of immune enteritis in the stage of neoadjuvant therapy. These findings suggested that we should pay much attention to the emergence of immune-related AEs, especially those uncommon AEs during treatment.

Studies conducted in patients with ESCC receiving neoadjuvant concurrent chemoradiotherapy showed that 18 of the 37 patients achieved pCR, and the rate of pCR was $49 \%$, while the occurrence of AEs $\geq$ grade 3 was as high as $25 \%[3]$. Patients whose tumors achieved pCR to neoadjuvant therapy had significantly longer relapse-free survival and overall survival[15]. Since the factors influencing therapeutic regimens for esophageal cancer between Eastern and Western countries are different, therapeutic strategies adopted in Asian countries, especically in Japan and China, often differ from those in Western countries[16]. The JCOG9907 study compared the clinical efficacy of neoadjuvant chemotherapy and adjuvant chemotherapy in patients with stage II/III ESCC, the results showed that the 5-year survival rate of patients who received neoadjuvant chemotherapy was significantly higher than that of those who received adjuvant chemotherapy.There was no increase of postoperative complications and hospital mortality $[4,5]$. Therefore, at present in Japan, patients with stage II/III ESCC are recommended for neoadjuvant chemotherapy followed by surgery or simultaneous chemoradiotherapy. In China, there was no standard regimen for patients with stage II/III ESCC, the recommended regimen for this kind of patients is to enroll in clinical studies.

In China a randomized clinical trial in patients with locally advanced ESCC comparing the neoadjuvant chemoradiotherapy to neoadjuvant chemotherapy showed that the pCRs were $35.7 \%$ and $3.8 \%$ individually, and there was no significant difference in postoperative morbidity between the two groups (47.4\% vs 42.6\%)[17]. The pCR rate of neoadjuvant chemotherapy in the Chinese population with locally advanced ESCC is relatively low and needs improvement. If the performance status of patients is good, simultaneous chemoradiotherapy might be a good choice. With the development of immunotherapy, clinical trials based on immunotherapy are also conducted in a neoadjuvant setting in such patients. In PALACE-1 study, pembrolizumab was added in chemoradiotherapy for ESCC, the rate of pCR was $55.6 \%$ (10/18), grade III and higher AEs occurred in 13 patients (13/20,65\%), and one patient died[18]. Simultaneously, the addition of atezolizumab in neoadjuvant chemoradiotherapy in patients with resectable esophageal adenocarcinoma showed that the rate of pCR was $25 \%$, grade $3 / 4$ AEs was observed in 40\% (16/40) patients and one patient died[19]. The incidence of grade III and higher AEs in patients with locally advanced ESCC treated with neoadjuvant concurrent chemoradiotherapy combined with anti-PD-1/PD-L1 antibodies was high. The neoadjuvant therapeutic regimen containing immunotherapy in patients with locally advanced ESCC needs to be optimized. In Chinese patients, neoadjuvant chemoimmunotherapy might be one of the choices $[4,5,17,18]$.

When the anti-PD-1 antibody is combined with other kinds of therapies such as chemotherapy, radiotherapy, anti-angiogenesis, or immunomodulator, the sequence of anti-PD-1 antibody and other regimens might influence the clinical efficacy[7, 20-22]. The TONIC trial was a non-comparative phase II study to explore whether induction treatment could induce a more inflamed tumor microenvironment. Sixty-seven patients with metastatic triple-negative breast cancer were randomized to nivolumab without 
induction or with 2-week low-dose induction with irradiation or chemotherapy, the results showed that the ORRs in the doxorubicin cohort, cisplatin cohort, cyclophosphamide corhort, radiotherapy cohort, and no induction cohort were $35 \%, 23 \%, 8 \%, 8 \%$, and $17 \%$, respectively. Immune-related genes involved in PD1/PD-L1 and T cell cytotoxicity pathways were upregulated in the doxorubicin and cisplatin cohorts[22]. These results indicated that preconditioning with some chemotherapeutic agents could induce a more inflamed tumor microenviroment which would favor of immunotherapy. GeparNuevo study was a phase II study to investigate whether the addition of durvalumab into neoadjuvant chemotherapy increases the pCR rate in patients with early triple-negative breast cancer, the patients were divided into three cohorts, the pCR rate with durvalumab was $53.4 \%$ versus placebo $44.2 \%(P=0.287)$, while in the window-phase cohort the pCR rate was $61 \%$ versus $41.4 \%(P=0.048)$ [21]. This study showed again that preconditioning with durvalumab 2 weeks before chemoimmunotherapy could improve the pCR rate,which showed the importance of administration sequence of immunotherapy and chemotherapy. Therefore, when designing a chemoimmunotherapy regimen, the doses, sequence, and administration intervals should be considered. Considering the mechanisms of synergy between chemotherapy and immunotherapy, sequential approaches might be helpful[7].

The mechanisms of postponing the application of anti-PD-1 antibody for 2 days when combined with chemotherapy with anti-PD-1 antibody may be as follows: studies in preclinical animal models found that cyclophosphamide could upregulate PD-1 and PD-L1 expression on tumor-specific CD8 + T cells in tumorbearing C3 mice.Then the application of anti-PD-1 treatment could increase the anti-tumor effect, thus increasing the systemic anti-tumor effect[23]. Short-term chemotherapy in patients with triple negative breast cancer may induce a more favorable tumor microenvironment and increase the likelihood of response to PD-1 blockade therapy[21]. In lung squamous cell cancer and colon cancer, there is a synergistic effect induced by low dose chemotherapy followed by anti-PD-1 antibodies[24, 25]. Therefore, theoretically, the effect of using PD-1 antibodies after chemotherapy may be better than that of using them simultaneously, but this does not imply that the later use of an anti-PD-1 antibody might yield better efficacy. Studies in patients with brain metastasis of melanoma have found that the immunotherapeutic efficacy within 4 weeks after stereotactic radiosurgery treatment is better than that after 4 weeks[20]. The subgroup analysis of the PERCIFIC study also showed that the efficacy of anti-PD-L1 was better in the group in which anti-PD-L1 was applied within 2 weeks after the end of simultaneous chemoradiotherapy[26]. A clinical study showed that the earlier application of anti-PD-1 antibody the better[10]. Therefore, we postpone the application of anti-PD-1 antibody for 2 days after chemotherapy. When the anti-PD-1 antibody was applied alone, the T cells with anti-tumor activity in peripheral blood reached the peak 1 or 2 weeks after the first dose of anti-PD-1 antibody, and then decreased gradually[27]. To reduce the influence of the second cycle of chemotherapy on activated T cells, the immunechemotherapy cycle in the experimental group was 3-4 weeks. In this study, a trend of higher pCR rate was observed in the experimental cohort than that in control cohort $\left(\chi^{2}=3.092, P=0.079\right)$, indicating the importance of the sequence of chemotherapy and immunotherapy. 
Thus far, there were no biomarkers for predicting the efficacy of anti-PD-1 therapy in esophageal cancer, although PD-L1 expression has been used to guide anti-PD-1 therapy in non-small cell lung cancer without driver gene mutations[28]. In KEYNOTE 590 study, patients with PD-L1 CPS $₫ 10$ could benefit more from chemotherapy combined with pembrolizumab, while the risk of death in patients with CPS $<10$ could also be reduced[6]. Patients could benefit from anti-PD-1 therapy regardless of the expression of PD-L1 in ATTRACTION-3 and ESCORT studies[14, 29]. In this study, we examined the expression of PD-L1 CPS in 14 patients (7 in each group) with the samples before treatment, the results showed that almost all the patients had PD-L1 CPS $\leq 1$ (except one with PD-L1 CPS 10), which indicated that our patients might represent the group of patients insensitive to anti-PD-1 therapy. Two of the 6 patients with PD-L1 CPS $\leq 1$ in the experimental cohort achieved pCR, and the rate of $\mathrm{PCR}$ in the experiment group was $36.4 \%$, it might be even higher if we could avoid the influence of COVID-19. A higher rate of pCR might be obtained by delaying anti-PD-1 antibody application by 2 days when chemotherapy plus anti-PD-1 therapy were used.

There are several limitations of this study. First was the influence of COVID-19 from February to May in 2020. The chemoimmunotherapy cycles were forcibly prolonged, and the operations were postponed in several patients, which may be one of the reasons that the rate of pCR in this study was lower than that reported previously[18, 30-32]. Second, as this study was a small sample exploratory study, we initially planned to enroll 30 patients. Because the representation of the small sample was not strong, bias was inevitable in the process of selecting patients. The PD-L1 expression results showed that only one patient with PD-L1 CPS was 10, the others were PD-L1 $\leq 1$, which suggested that the patients in this study may only present the group of patients insensitive to anti-PD-1 therapy. Thirdly, due to the limitation of the preoperative specimens, not all the patients have enough tissue specimens for PD-L1 CPS examination. Lastly, there was no exploratory study on other biomarkers that might affect the efficacy. However, our results showed that the PCR rate in patients with delayed application of anti-PD- 1 antibody was $36.4 \%$. There was a statistical boundary difference, suggesting that when chemotherapy combined with anti-PD1 antibody was used in a neoadjuvant setting, delaying anti-PD-1 antibody therapy by 2 days might have greater clinical benefits.

\section{Conclusions}

Our results showed that neoadjuvant chemotherapy plus toripalimab is safe and effective in patients with locally advanced ESCC, with a pCR rate of $20.8 \%$. In the process of neoadjuvant chemotherapy combined with anti-PD-1 antibody, the application of anti-PD-1 antibody 2 days after chemotherapy maybe more conducive to the synergistic effects. Further clinical studies are needed in a larger sample of patients to verify the influence of different administration sequences on $\mathrm{PCR}$ and explore its underlying biomarkers. We are conducting a phase III study to compare the clinical efficacy of neoadjuvant chemoimmunotherapy to neoadjuvant chemotherapy for resectable esophageal cancer (NCT 04280822).

\section{List Of Abbreviations}


pCR

pathological complete response; ESCC:esophageal squamous cell carcinoma; CPS:combined positive score; CT:computed tomography; MRI:magnetic resonance imaging; AE:adverse events; $\mathrm{NCI}$-CTC:National Cancer Institute Common Terminology Criteria; ORR:objective response rate;

\section{Declarations}

\section{Ethics approval and consent to participate}

This study was approved by Henan Cancer Hospital Ethics Committee and the approval number was 2019205. All the participants agree to take part in this study according to the ethical guidelines of Declaration of Helsinki and signed an informed consent for samples and anonymized information to be used.

\section{Consent for publication}

All the authors have seen and approved the manuscript and consent publication.

\section{Availability of data and materials}

The data that support the findings of this study are available on request from the corresponding author (Q.G.). The data are not publicly available as they contain information that could compromise the privacy of the research participants.

\section{Competing interests}

The authors declare there are no conflicts of interest.

\section{Funding}

This study was supported by the National Natural Science Foundation of China (grant numbers 81902902 and 81972802).

\section{Authors' contributions}

Q.G. and J. Z. took part in the conceptualization. W.X., L.Z., Y.Z., and L. L. carried out writing-original draft preparation. Q. G., X. R, and Z. W. carried out writing-review and editing. T. L., L. H, and B. M. carried out the statistical analyses and visualization. X. C. and H. Z. performed the pathological evaluation and PDL1 examination. J. Q. and Y. Z. performed the imaging evaluation. B. L., X. L., G. L., D. Y., Y. Y., Y. S, and X. F. performed the data collection. All the authors read and approved the final manuscript.

\section{Acknowledgements}

We are thankful to the patients and their families for their cooperation, and to Editage (www.editage.cn) for English language editing. 


\section{References}

1. ChenW,ZhengR,BaadePD,ZhangS,ZengH,BrayF,etal.Cancer statistics in China, 2015.CA Cancer J Clin,2016.66(2):115 - 32.doi:10.3322/caac.21338

2. YangH,LiuH,ChenY,ZhuC,FangW,YuZ,etal.Neoadjuvant Chemoradiotherapy Followed by Surgery Versus Surgery Alone for Locally Advanced Squamous Cell Carcinoma of the Esophagus (NEOCRTEC5010): A Phase III Multicenter, Randomized, Open-Label Clinical Trial.J Clin Oncol,2018.36(27):2796-2803.doi:10.1200/JC0.2018.79.1483

3. vanHagenP,HulshofMC,vanLanschotJJ,SteyerbergEW,vanBerge HenegouwenMI,WijinhovenBP,etal.Preoperative chemoradiotherapy for esophageal or junctional cancer.N Engl J Med,2012.366(22):2074-84.doi:10.1056/NEJMoa1112088

4. AndoN,KatoH,IgakiH,ShinodaM,OzawaS,ShimizuH,etal.A randomized trial comparing postoperative adjuvant chemotherapy with cisplatin and 5-fluorouracil versus preoperative chemotherapy for localized advanced squamous cell carcinoma of the thoracic esophagus (JCOG9907).Ann Surg Oncol,2012.19(1):68-74.doi:10.1245/s10434-011-2049-9

5. HiraoM,AndoN,TsujinakaT,UdagawaH,YanoM,YamanaH,etal.Influence of preoperative chemotherapy for advanced thoracic oesophageal squamous cell carcinoma on perioperative complications. $\mathrm{Br} \mathrm{J}$ Surg,2011.98(12):1735-41.doi:10.1002/bjs.7683

6. Kato K, Sun J, Shah MA, Enzinger PC, Adenis A, Doi T, et al. Pembrolizumab plus chemotherapy versus chemotherapy as first-line therapy in patients with advanced esophageal cancer: The phase 3 KEYNOTE-590 study. Ann Oncol. 2020;31(Suppl_4):1142-215.

7. Salas-BenitoD,Perez-GraciaJL,Ponz-SarviseM,Rodriguez-RuizME,MartinezForerol,CastanonE,etal.Paradigms on Immunotherapy Combinations with Chemotherapy.Cancer Discov,2021.doi:10.1158/2159-8290.CD-20-1312

8. ShitaraK,VanCutsemE,BangYJ,FuchsC,WywiczL,LeeKW,etal.Efficacy and Safety of Pembrolizumab or Pembrolizumab Plus Chemotherapy vs Chemotherapy Alone for Patients With First-line, Advanced Gastric Cancer: The KEYNOTE-062 Phase 3 Randomized Clinical Trial.JAMA Oncol,2020.6(10):1571-1580.doi:10.1001/jamaoncol.2020.3370

9. MessenheimerDJ,JensenSM,AfentoulisME,WegmannKW,FengZ,FriedmanDJ,etal.Timing of PD-1 Blockade Is Critical to Effective Combination Immunotherapy with Anti-OX40.Clin Cancer Res,2017.23(20):p.6165-6177.doi:10.1158/1078-0432.CCR-16-2677

10. TopalianSL,TaubeJM,PardollDM.Neoadjuvant checkpoint blockade for cancer immunotherapy.Science,2020.367(6477).doi:10.1126/science.aax0182

11. QuJ,ShenC,QinJ,WangZ,LiuZ,GaoJ,etal.The MR radiomic signature can predict preoperative lymph node metastasis in patients with esophageal cancer.Eur Radiol,2019.29(2):906914.doi:10.1007/s00330-018-5583-z

12. RiceTW,IshwaranH,FergusonMK,BlackstoneEH,GoldstrawP.Cancer of the Esophagus and Esophagogastric Junction: An Eighth Edition Staging Primer.J Thorac Oncol,2017.12(1):36- 
42.doi:10.1016/j.jtho.2016.10.016

13. ZhangX,JainD.Updates in staging and pathologic evaluation of esophageal carcinoma following neoadjuvant therapy.Ann N Y Acad Sci,2020.1482(1):163-176.doi:10.1111/nyas.14462

14. KatoK,ChoBC,TakahashiM,OkadaM,LinCY,ChinK,etal.Nivolumab versus chemotherapy in patients with advanced oesophageal squamous cell carcinoma refractory or intolerant to previous chemotherapy (ATTRACTION-3): a multicentre, randomised, open-label, phase 3 trial.Lancet Oncol,2019.20(11):1506-1517.doi:10.1016/S1470-2045(19)30626-6

15. Fernandez-MartinezA,KropIE,HillmanDW,PolleyMY,ParkerJ,HuebnerL,etal.Survival, pathologic response, and genomics in CALGB 40601 (Alliance), a neoadjuvant phase III trial of paclitaxeltrastuzumab with or without lapatinib in Her2-positive breast cancer.J Clin Oncol.2020.38(35):41844193.doi:10.1200/JC0.20.01276

16. KoyanagiK,KanamoriK,NinomiyaY,YatabeK,HiguchiT,YamamotoM,etal.Progress in Multimodal Treatment for Advanced Esophageal Squamous Cell Carcinoma: Results of Multi-Institutional Trials Conducted in Japan.Cancers (Basel),2020.13(1).doi:10.3390/cancers13010051

17. WangH,TangH,FangY,TanL,YinJ,ShenY,etal.Morbidity and Mortality of Patients Who Underwent Minimally Invasive Esophagectomy After Neoadjuvant Chemoradiotherapy vs Neoadjuvant Chemotherapy for Locally Advanced Esophageal Squamous Cell Carcinoma: A Randomized Clinical Trial.JAMA Surg,2021.doi:10.3390/cancers13010051

18. LiC,ZhaoS,ZhengY,HanY,ChenX,ChengZ,etal.Preoperative pembrolizumab combined with chemoradiotherapy for oesophageal squamous cell carcinoma (PALACE-1).Eur J Cancer,2020.144:232-241.doi:10.1016/j.ejca.2020.11.039

19. van denEndeT,ClercqN,vanBerge HenegouwenMI,GisbertzSS,GeijsenD,VerhoevenR,etal.Neoadjuvant Chemoradiotherapy Combined with Atezolizumab for Resectable Esophageal Adenocarcinoma: A Single Arm Phase II Feasibility Trial (PERFECT).Clin Cancer Res,2021.doi:10.1158/1078-0432.CCR20-4443

20. QianJM,YuJB,KlugerHM,ChiangVL.Timing and type of immune checkpoint therapy affect the early radiographic response of melanoma brain metastases to stereotactic radiosurgery.Cancer,2016.122(19):3051-8.doi:10.1002/cncr.30138

21. LoibIS,UntchM,BurchardiN,HuoberJ,SinnBV,BlohmerJU,etal.A randomised phase II study investigating durvalumab in addition to an anthracycline taxane-based neoadjuvant therapy in early triple-negative breast cancer: clinical results and biomarker analysis of GeparNuevo study.Ann Oncol,2019.30(8):1279-1288.doi:10.1093/annonc/mdz158

22. VoorwerkL,SlagterM,HorlingsHM,SikorskaK,van deVijverKK,deMaakerM,etal.Immune induction strategies in metastatic triple-negative breast cancer to enhance the sensitivity to PD-1 blockade: the TONIC trial.Nat Med,2019.25(6):920-928.doi:10.1038/s41591-019-0432-4

23. WeirGM,HrytsenkoO,QuintonT,BerinsteinNL,StanfordMM,MansourM.Anti-PD-1 increases the clonality and activity of tumor infiltrating antigen specific $T$ cells induced by a potent immune therapy 
consisting of vaccine and metronomic cyclophosphamide.J Immunother Cancer,2016.4:68.doi:10.1186/s40425-016-0169-2

24. HeX,DuY,WangZ,WangX,DuanJ,WanR,etal.Upfront dose-reduced chemotherapy synergizes with immunotherapy to optimize chemoimmunotherapy in squamous cell lung carcinoma.J Immunother Cancer,2020.8(2).doi:10.1136/jitc-2020-000807

25. FuD,WuJ,LaiJ,LiuY,ZhouL,ChenL,etal.T cell recruitment triggered by optimal dose platinum compounds contributes to the therapeutic efficacy of sequential PD-1 blockade in a mouse model of colon cancer.Am J Cancer Res,2020.10(2):473-490.

26. AntoniaSJ,VillegasA,DanielD,VicenteD,MurakamiS,HuiR,etal.Overall Survival with Durvalumab after Chemoradiotherapy in Stage III NSCLC.N Engl J Med,2018.379(24):23422350.doi:10.1056/NEJMoa1809697

27. FordePM,ChaftJE,SmithKN,AnagnostouV,CottrellTR,HellmannMD,etal.Neoadjuvant PD-1 Blockade in Resectable Lung Cancer.N Engl J Med,2018.378(21):1976-1986.doi:10.1056/NEJMoa1716078

28. ReckM,Rodriguez-AbreuD,BobinsonAG,HuiR,CsosziT,FulopA,etal.Pembrolizumab versus Chemotherapy for PD-L1-Positive Non-Small-Cell Lung Cancer.N Engl J Med,2016.375(19):18231833.doi:10.1056/NEJMoa1606774

29. HuangJ,XuJ,ChenY,ZhuangW,ZhangY,ChenZ,etal.Camrelizumab versus investigator's choice of chemotherapy as second-line therapy for advanced or metastatic oesophageal squamous cell carcinoma (ESCORT): a multicentre, randomised, open-label, phase 3 study.Lancet Oncol,2020.21(6):832-842.doi:10.1016/S1470-2045(20)30110-8

30. ShapiroJ,vanLanschotJJJB,HulshofMccm,vanHagenP,vanBerge HenegouwenMI,WijnhovenBPL,etal.Neoadjuvant chemoradiotherapy plus surgery versus surgery alone for oesophageal or junctional cancer (CROSS): long-term results of a randomised controlled trial.Lancet Oncol,2015.16(9):1090-1098.doi:10.1016/S1470-2045(15)00040-6

31. KelsenDP,WinterKA,GundersonLL,MortimerJ,EstesNC,HallerDG,etal.Long-term results of RTOG trial 8911 (USA Intergroup 113): a random assignment trial comparison of chemotherapy followed by surgery compared with surgery alone for esophageal cancer.J Clin Oncol,2007.25(24):371925.doi:10.1200/JCO.2006.10.4760

32. AhmedN,OwenJ,AbdalmassihM,KhanJ,NugentZ,QingG,etal.Outcome of Locally Advanced Esophageal Cancer Patients Treated With Perioperative Chemotherapy and Chemoradiotherapy Followed by Surgery.Am J Clin Oncol,2021.44(1):10-17.doi:10.1097/COC.0000000000000773

\section{Figures}




\section{0 patients enrolled and randomized}

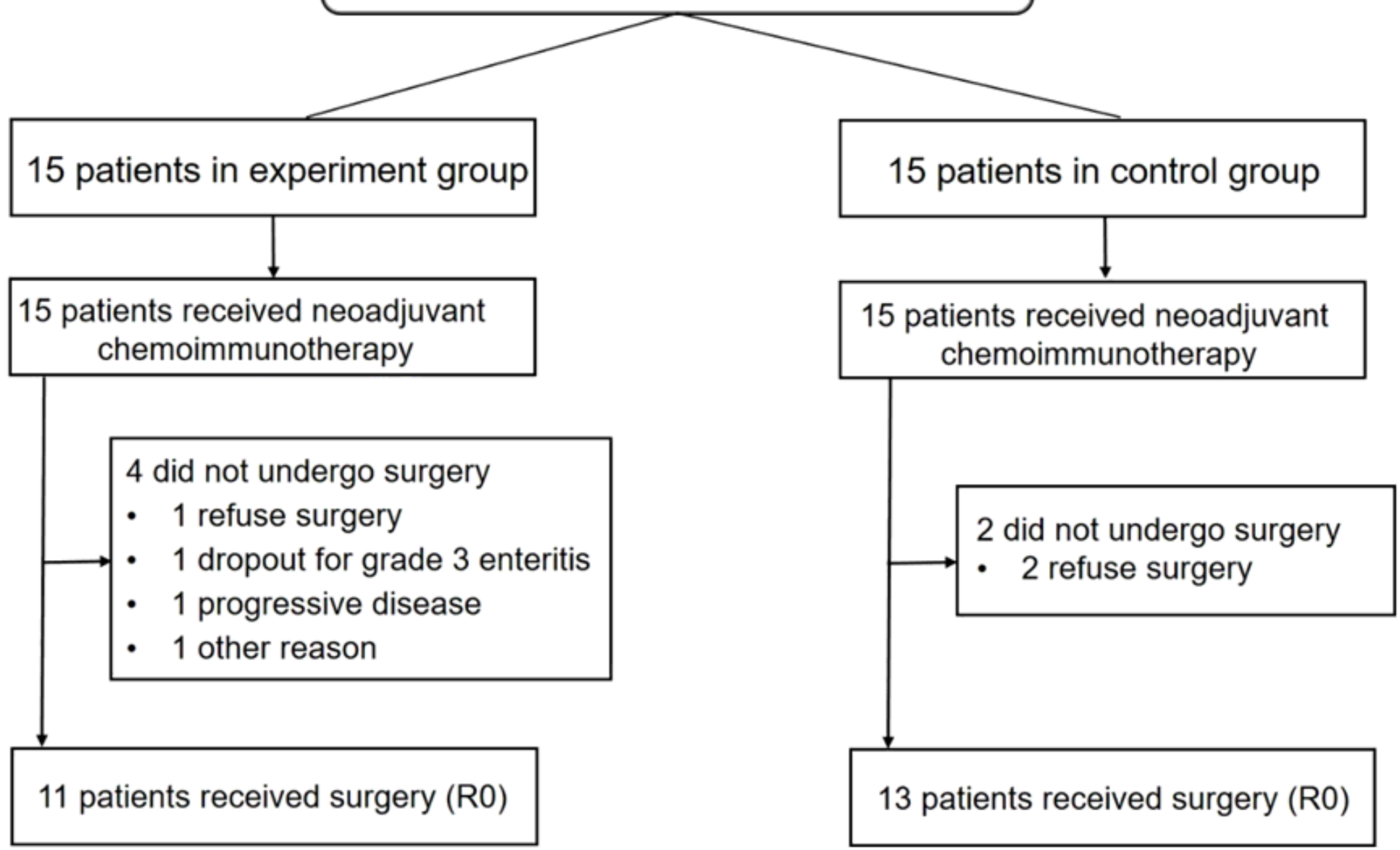

Figure 1

CONSORT Diagram 


\section{Before neoadjuvant}
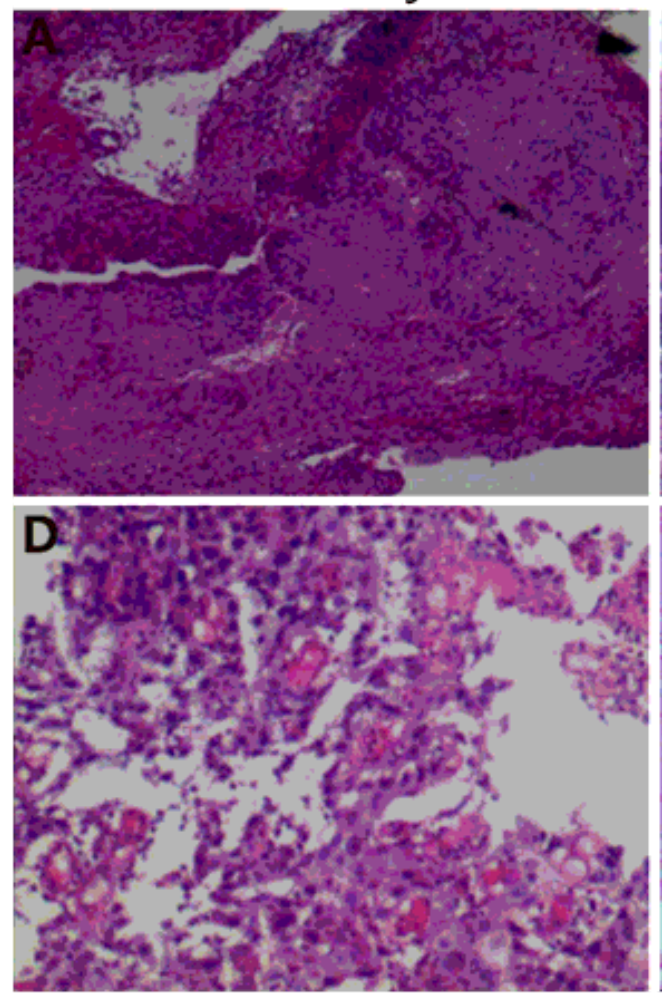

After neoadjuvant
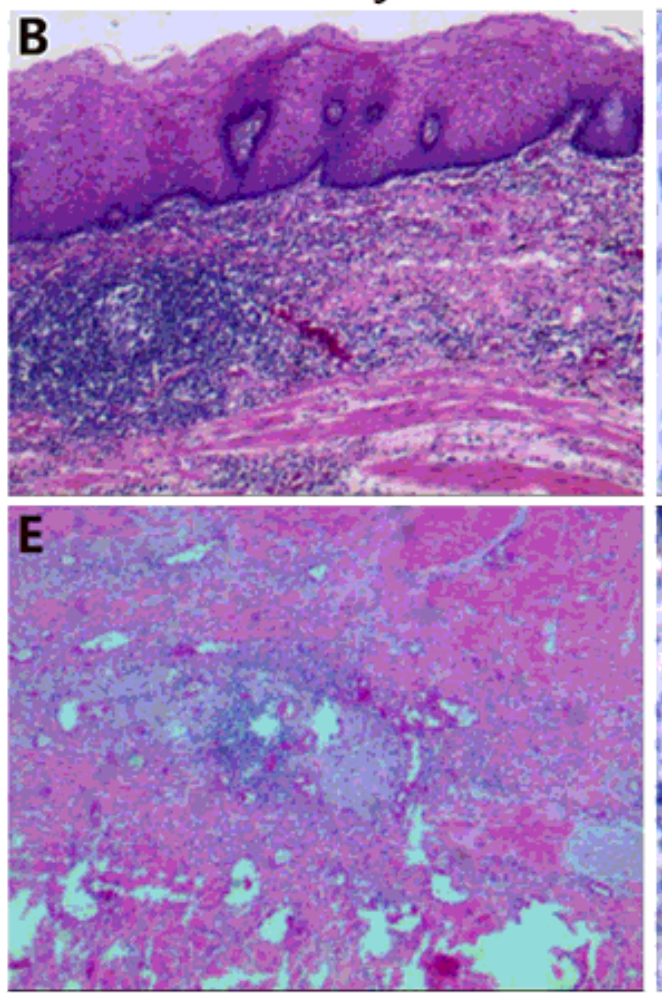

PD-L1 CPS
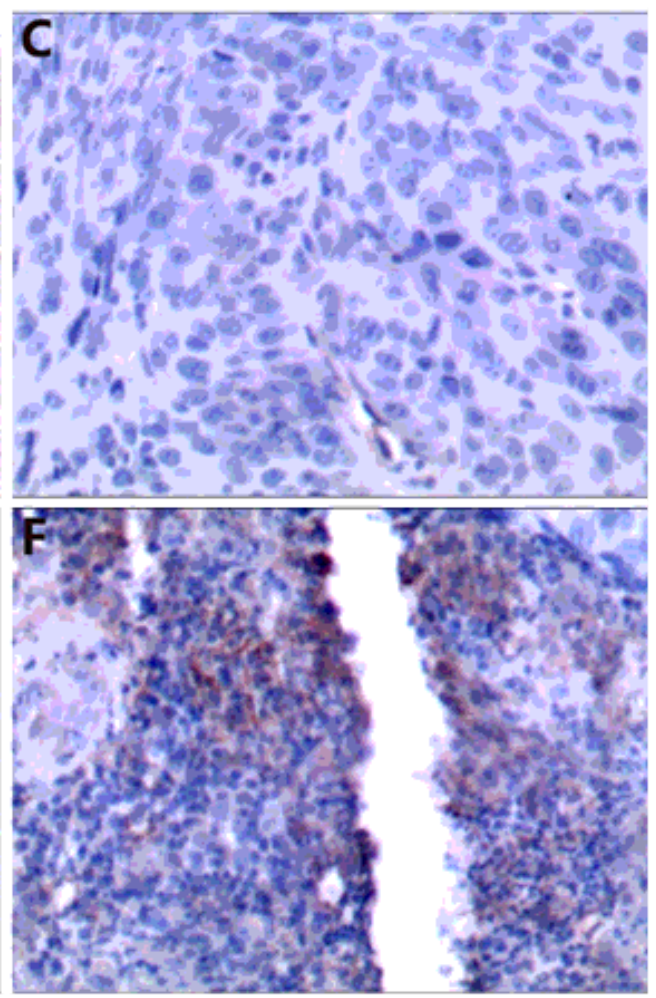

\section{Figure 2}

The pathological changes before and after neoadjuvant chemoimmunotherapy and the PD-L1 expression. A and B show the patholgocal changes in patient 1, after neoadjuvant chemoimmunotherapy, the tumor cells disappeared and many lymphocytes infiltrated. $C$ shows the expression of PD-L1 CPS in the sample before therapy in patient 1, and the expression of PD-L1 CPS was lower than 1. D and E show the pathological changes in patient 4 , after neoadjuvant chemoimmunotherapy, the tumor cells disappeared and some lymphocytes infiltrated.F shows the expression of PD-L1 CPS in the sample before therapy in patient 4, and the expression of PD-L1 CPS was 10. 
Before neoadjuvant
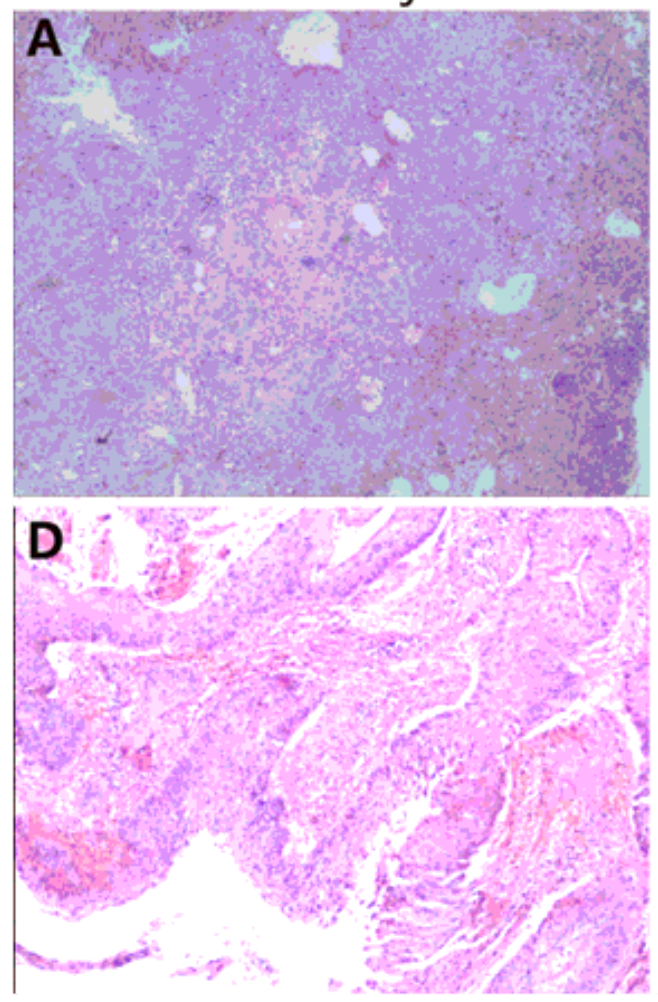

After neoadjuvant
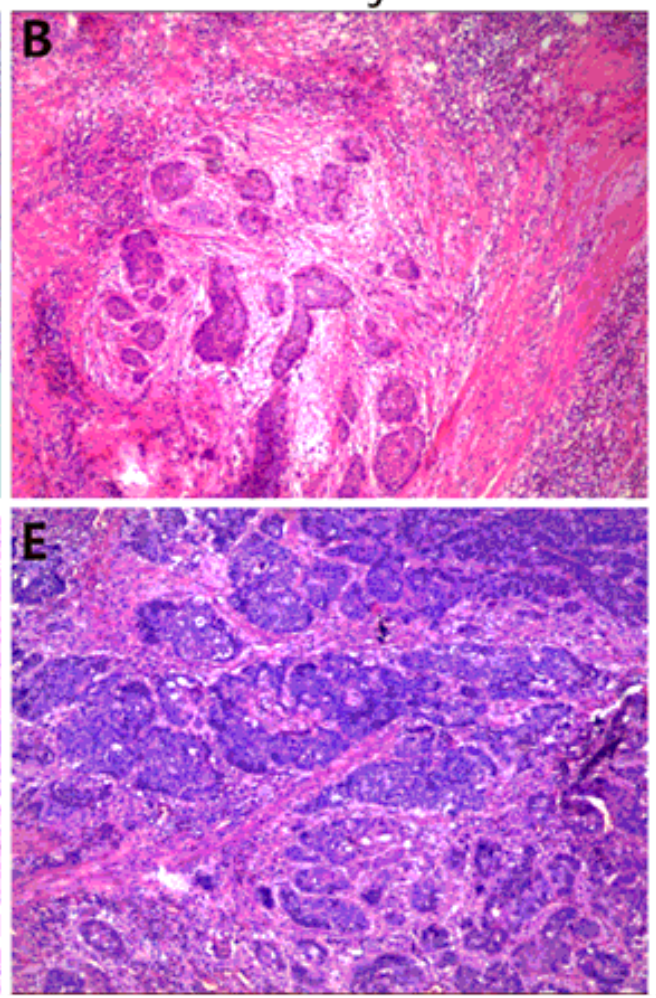

PD-L1 CPS
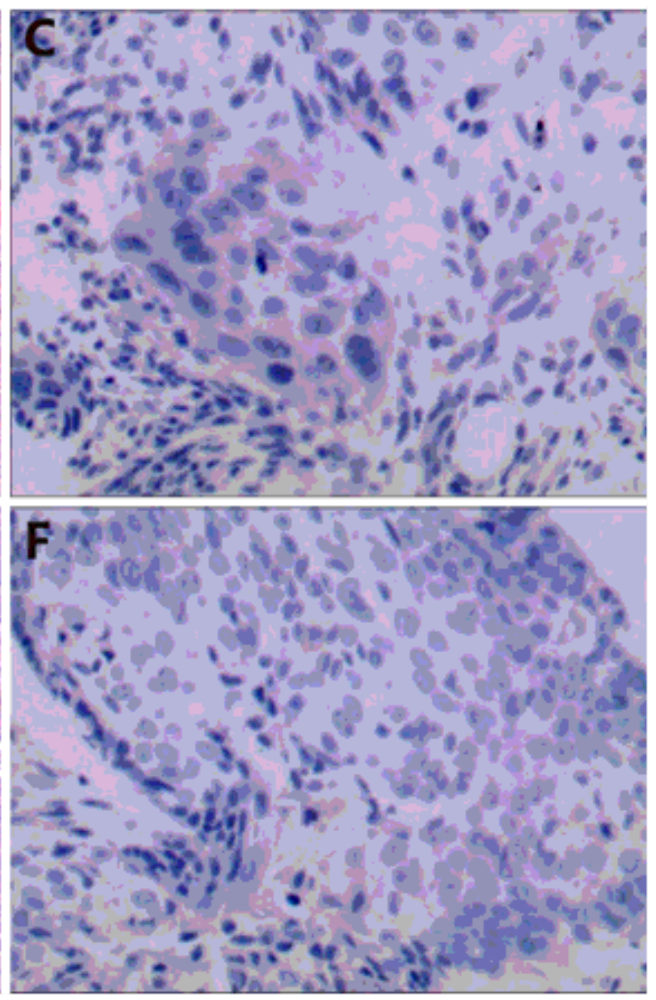

\section{Figure 3}

The pathological changes before and after neoadjuvant chemoimmunotherapy and the PD-L1 expression. A and B show the pathological changes in patient 7 , after neoadjuvant chemoimmunotherapy, there are a large number of tumor cells left in the section and few lymphocytes infiltrated. C shows the expression of PD-L1 CPS in the sample before therapy in patient 7, and the expression of PD-L1 CPS was lower than 1. D and E show the pathological changes in patient 8 , after neoadjuvant chemoimmunotherapy, there are a large number of tumor cells left in the section and few lymphocytes infiltrated. F shows the expression of PD-L1 CPS in the sample before therapy in patient 8 , and the expression of PD-L1 CPS was lower than 1.

\section{Supplementary Files}

This is a list of supplementary files associated with this preprint. Click to download.

- supplementarydata.doc 